\title{
1,1,2,2,3,3,4,4,4-Nonafluorobutane-1-sulfonyl fluoride (NfF)
}

\section{Timon Kurzawa}

Institut für Chemie und Biochemie, Freie Universität Berlin, Takustr. 3, 14195 Berlin, Germany

kurzimon@chemie.fu-berlin.de

\section{Introduction}

1,1,2,2,3,3,4,4,4-Nonafluorobutane-1-sulfonyl fluoride (NfF) is a versatile compound in organic synthesis. It can be used as a fluoride source for the nucleophilic introduction of fluorine, ${ }^{1}$ but it is also frequently applied as sulfonylation reagent generating intermediates with strong electronwithdrawing perfluorinated alkyl substituents. ${ }^{2}$
Timon Kurzawa was born in Berlin in 1988. He prepared his Bachelor thesis in the group of Prof. Reissig and his M.Sc. under the guidance of Prof. Christmann at the Freie Universität Berlin. Currently, he is working towards his Ph.D. under the supervision of Prof. Koert in Marburg. His research interests focus on asymmetric catalysis and natural product synthesis.

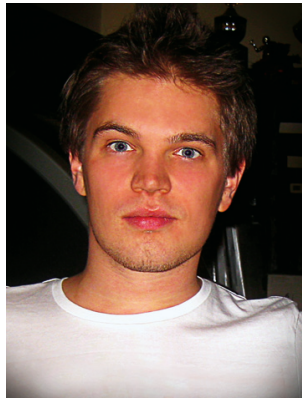

NfF is produced on industrial scale by anodic fluorination of sulfolene (1, Scheme 1$),{ }^{3}$ therefore it is a fairly cheap reagent and commercially available from several suppliers. The compound is bench-stable and storable for years, nontoxic and easy to handle.

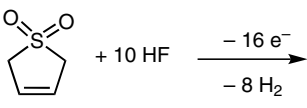

1

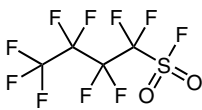

NfF
Scheme 1 Synthesis of NfF by anodic fluorination of sulfolene (1)

Table 1 Use of 1,1,2,2,3,3,4,4,4-Nonafluorobutane-1-sulfonyl fluoride (NfF)

(A) NfF can be used as a convenient fluoride source for the nucleophilic introduction of fluorine via the corresponding 0-nonaflate as demonstrated by Vorbrüggen. ${ }^{4}$ The application of this method on industrial scale was demonstrated for the synthesis of iodenosine by Takamatsu et al. ${ }^{5}$
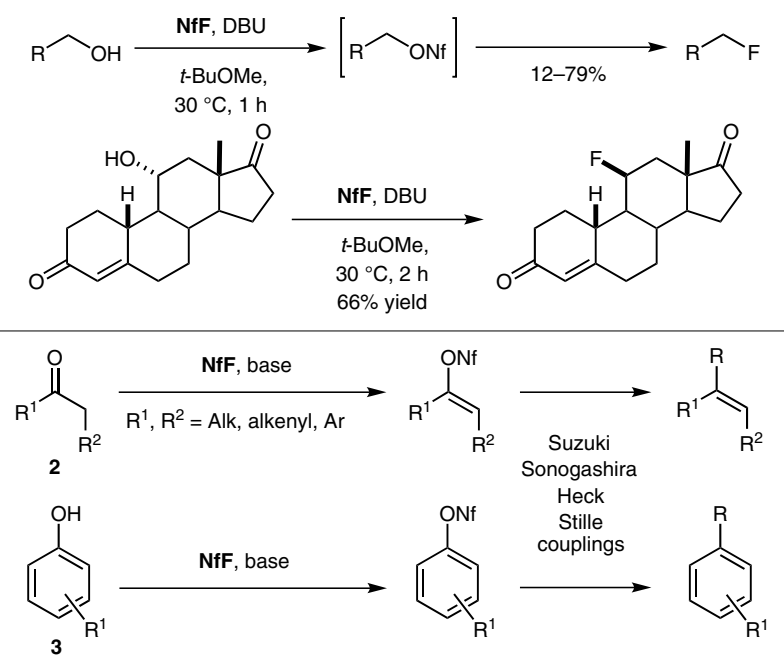

(B) NfF is also a common sulfonylating agent that allows the conversion of carbonyl compounds $\mathbf{2}$, their corresponding trimethylsilyl enol ethers or phenol derivatives $\mathbf{3}$ into nonaflates. ${ }^{6}$ These alkenyl and aryl nonaflates are efficient precursors for all kinds of crosscoupling reactions, in which they often show a better reactivity and a superior stability during transformations than the related tosylates or triflates. 
(C) The elimination of in situ formed alkenyl nonaflates derived from aldehydes or ketones in the presence of P-base gives terminal alkyne intermediates $\mathbf{4}$, as reported by Lyapkalo and Vogel in their one-pot synthesis of enynes $\mathbf{5}^{8}$ This synthetically useful method was later successfully applied by Huwyler and Carreira in their total synthesis of the chlorinated sesquiterpene $( \pm)$-gomerone $C,{ }^{9}$ or by Nicolaou et al. for the preparation of the cytotoxic polyketides myceliothermophins E, C and D. ${ }^{10}$

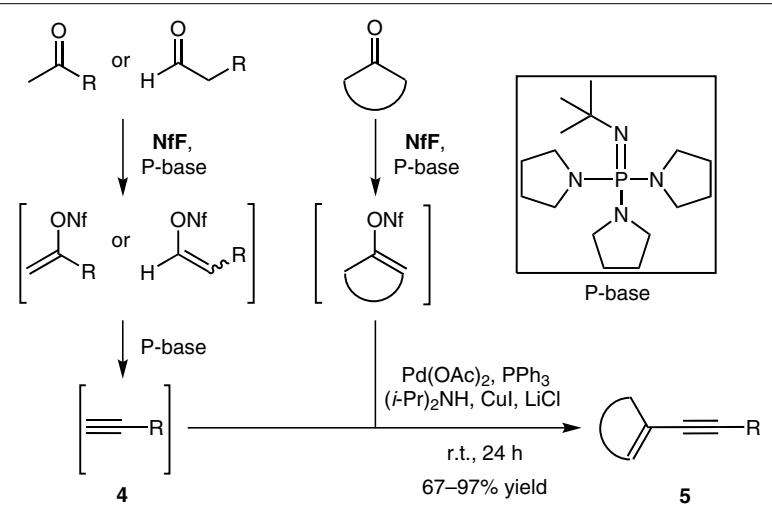

(D) Furthermore, NfF is also a powerful reagent in numerous nucleophilic displacement reactions under very mild reaction conditions. The intermediate nonaflates undergo intramolecular substitutions leading to useful compounds such as epoxides $\mathbf{6}(\mathrm{X}=\mathrm{O}),{ }^{11}$ aziridines $\mathbf{6}(\mathrm{X}=\mathrm{NTs})$ or thiazolines $\mathbf{7 .}{ }^{12}$

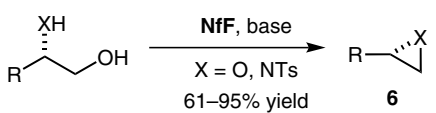

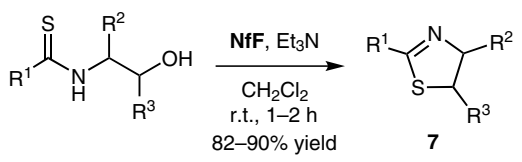

(E) Another interesting application of $\mathbf{N f F}$ involves its reaction with sodium azide, smoothly forming nonafluorobutane sulfonyl azide 8. ${ }^{13}$ This azide is a stable liquid at room temperature and therefore easier and safer to handle than the corresponding trifluoromethane sulfonyl azide. The compound was used for $\mathrm{Cu}(\mathrm{II})$-catalyzed diazo transfer reactions furnishing organic azides $(\mathrm{R}=$ alkyl or aryl $) .{ }^{14}$

\section{References}

(1) Vorbrüggen, H. Synthesis 2008, 1165.

(2) Zimmer, R.; Webel, M.; Reissig, H.-U. J. Prakt. Chem. 1998, 340, 274.

(3) Beyl, V.; Niederprüm, H.; Voss, P. Liebigs Ann. Chem. 1970, 731, 58.

(4) Bennua-Skalmowski, B.; Vorbrüggen, H. Tetrahedron Lett. 1995, 36, 2611.

(5) Takamatsu, S.; Katayama, S.; Hirose, N.; De Cock, E.; Schelkens, G.; Demillequand, M.; Brepoels, J.; Izawa, K. Nucleosides Nucleotides 2002, 21, 849.

(6) Hirsch, E.; Hünig, S.; Reissig, H.-U. Chem. Ber. 1982, 115, 3687.

(7) Högermeier, J.; Reissig, H.-U. Adv. Synth. Catal. 2009, 351, 2747.

(8) Lyapkalo, I. M.; Vogel, M. A. K. Angew. Chem. Int. Ed. 2006, 45, 4019.
(9) Huwyler, N.; Carreira, E. M. Angew. Chem. Int. Ed. 2012, 51, 13066.

(10) Nicolaou, K. C.; Shi, L.; Lu, M.; Pattanayak, M. R.; Shah, A. A.; Ioannidou, H. A.; Lamani, M. Angew. Chem. Int. Ed. 2014, 53, 10970.

(11) Klar, U.; Neef, G.; Vorbrüggen, H. Tetrahedron Lett. 1996, 37, 7497.

(12) Yan, Z.; Guan, C.; Yu, Z.; Tian, W. Tetrahedron Lett. 2013, 54, 5788.

(13) Zhu, S.-Z. J. Chem. Soc., Perkin Trans. 1 1994, 2077.

(14) (a) Al-Harrasi, A.; Pfrengle, F.; Prisyazhnyuk, V.; Reissig, H.-U. Chem. Eur. J. 2009, 15, 11632. (b) Suárez, J. R.; Trastoy, B.; PérézOjeda, M. E.; Marín-Barrios, R.; Chiara, J. L. Adv. Synth. Catal. 2010, 352, 2515. 